\title{
Supporting Information Infrared Spectrum of Fulvenallene and Fulvenallenyl in Helium Droplets
}

\begin{abstract}
Alaina R. Brown, Joseph T. Brice, Peter R. Franke, and Gary E. Douberly*
Department of Chemistry, University of Georgia, Athens, GA 30602, USA

* Corresponding Author: Email: douberly@uga.edu Tele: 01-706-542-3857
\end{abstract}

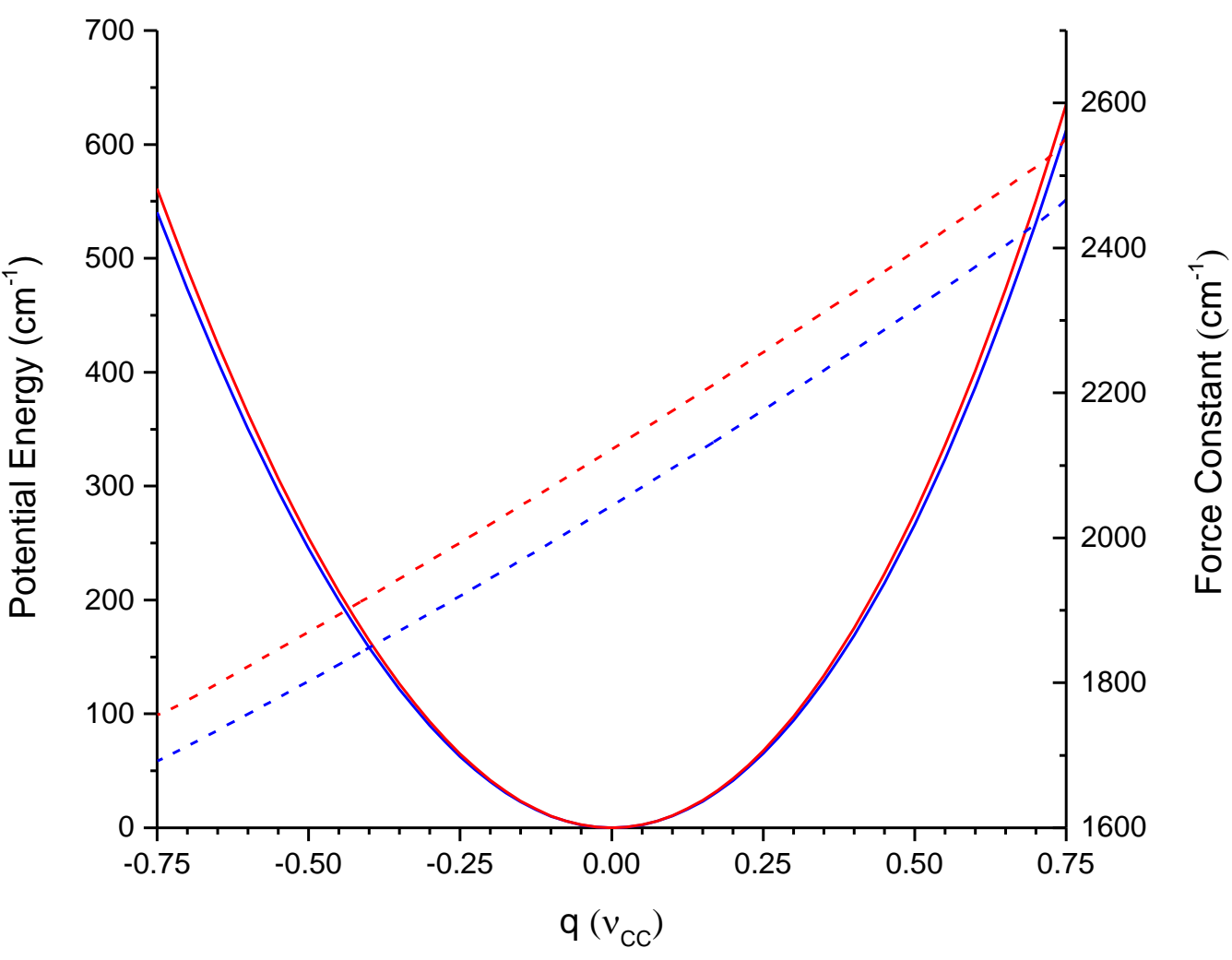

Figure S1. Plot of the CCSD(T)/ANO1 potential energy (primary Y-axis) using UHF (red) and ROHF (blue) reference wavefunctions. Displacements are made, from the corresponding equilibrium structures, in the UHF-CCSD(T)/ANO0 dimensionless normal coordinate associated with acetylenic $\mathrm{CC}$ bond stretching. The second derivatives of the potentials are plotted on the secondary Y-axis; these correspond roughly with the CCSD(T)/ANO1 harmonic frequencies when $\mathrm{q}=0$. 
Table S1. Selected Harmonic Frequencies of Ground State $\left(B_{1}\right)$ Fulvenallenyl Radical Obtained with Different Electronic Structure Methods and the ANO0 Basis Set.

\begin{tabular}{|c|c|c|c|}
\hline \multirow{2}{*}{$\begin{array}{c}\text { Approximate } \\
\text { Description }\end{array}$} & \multicolumn{3}{|c|}{ Harmonic Frequency $\left(\mathbf{c m}^{-1}\right)$} \\
\cline { 2 - 4 } "Allylic" Ring Deform & ROHF-CCSD(T) & ROHF-CCSD & EOMIP-CCSD \\
\hline "Dienylic" Ring Deform & 1032.2 & 680.0 & 678.6 \\
\hline Ethynyl CC Stretch & 2052.7 & 1059.0 & 1059.5 \\
\hline $\begin{array}{c}\text { Antisymmetric CH stretch } \\
\text { (Lower Carbons) }\end{array}$ & 3232.7 & 3256.7 & 3263.9 \\
\hline $\begin{array}{c}\text { Symmetric CH stretch } \\
\text { (Out-of-Phase) }\end{array}$ & 3243.6 & 3267.1 & 3274.0 \\
\hline $\begin{array}{c}\text { Antisymmetric CH stretch } \\
\text { (Upper Carbons) }\end{array}$ & 3265.0 & 3288.4 & 3293.3 \\
\hline $\begin{array}{c}\text { Symmetric CH stretch (In- } \\
\text { Phase) }\end{array}$ & 3269.4 & 3292.9 & 3297.5 \\
\hline Acetylenic CH Stretch & 3459.9 & 3483.5 & 3485.8 \\
\hline
\end{tabular}




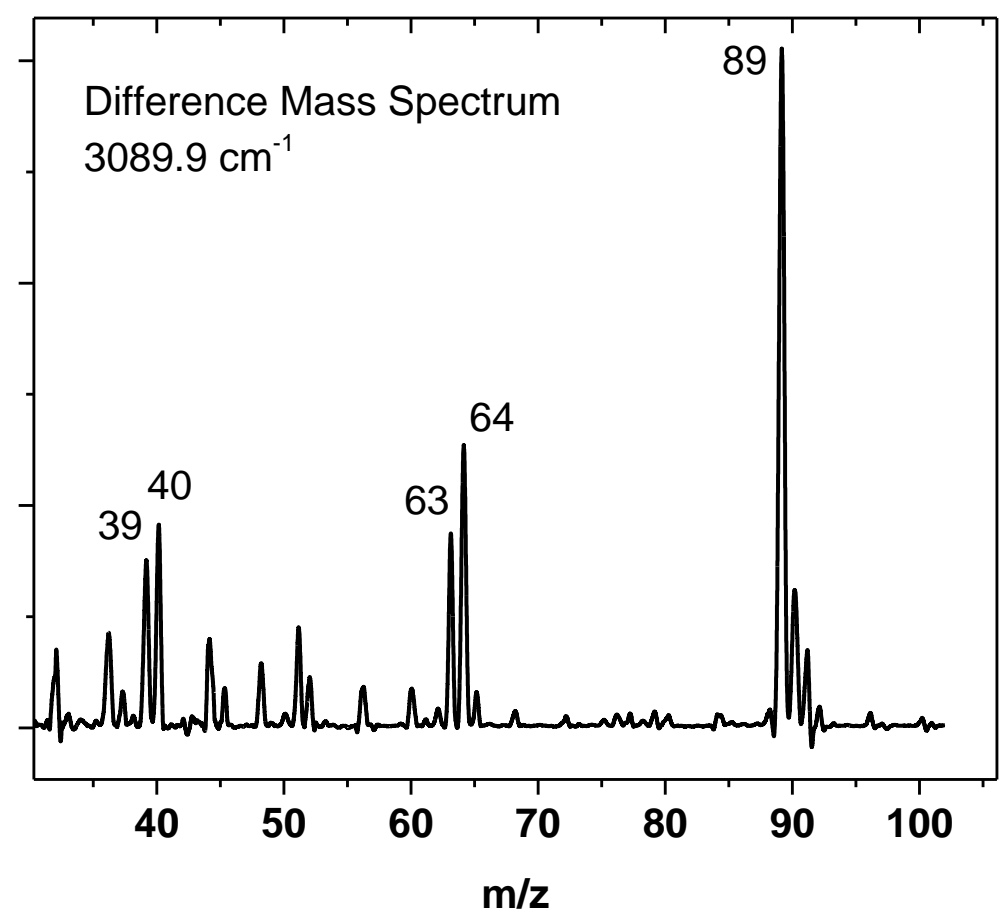

Figure S2. Difference depletion mass spectra measured with the laser frequency fixed to 3089.9 $\mathrm{cm}^{-1}$.

The mass spectrum of fulvenallene doped helium droplets can be obtained via a difference depletion mass spectrometry technique, the results from which are shown in Figure S2. With the pyrolysis source at room temperature, the IR laser is tuned to $3089.9 \mathrm{~cm}^{-1}$, i.e. the most intense resonant frequency for fulvenallene. The laser frequency is fixed and amplitude modulated at $80 \mathrm{~Hz}$ while the quadrupole mass spectrometer is slowly scanned across all mass channels. When processed with a lock-in amplifier, the resulting difference mass spectrum corresponds to the electron-impact ionization mass spectrum of only the subset of droplets that contain resonantly excited molecules. In Figure S2, mass channel 89 is by far the most intense, indicating that this channel carries the largest laser-induced ion depletion signal at $3089.9 \mathrm{~cm}^{-1}$ when the pyrolysis source is kept at room temperature, i.e. indicating that this is the largest mass channel associated with fulvenallene doped helium droplets. 


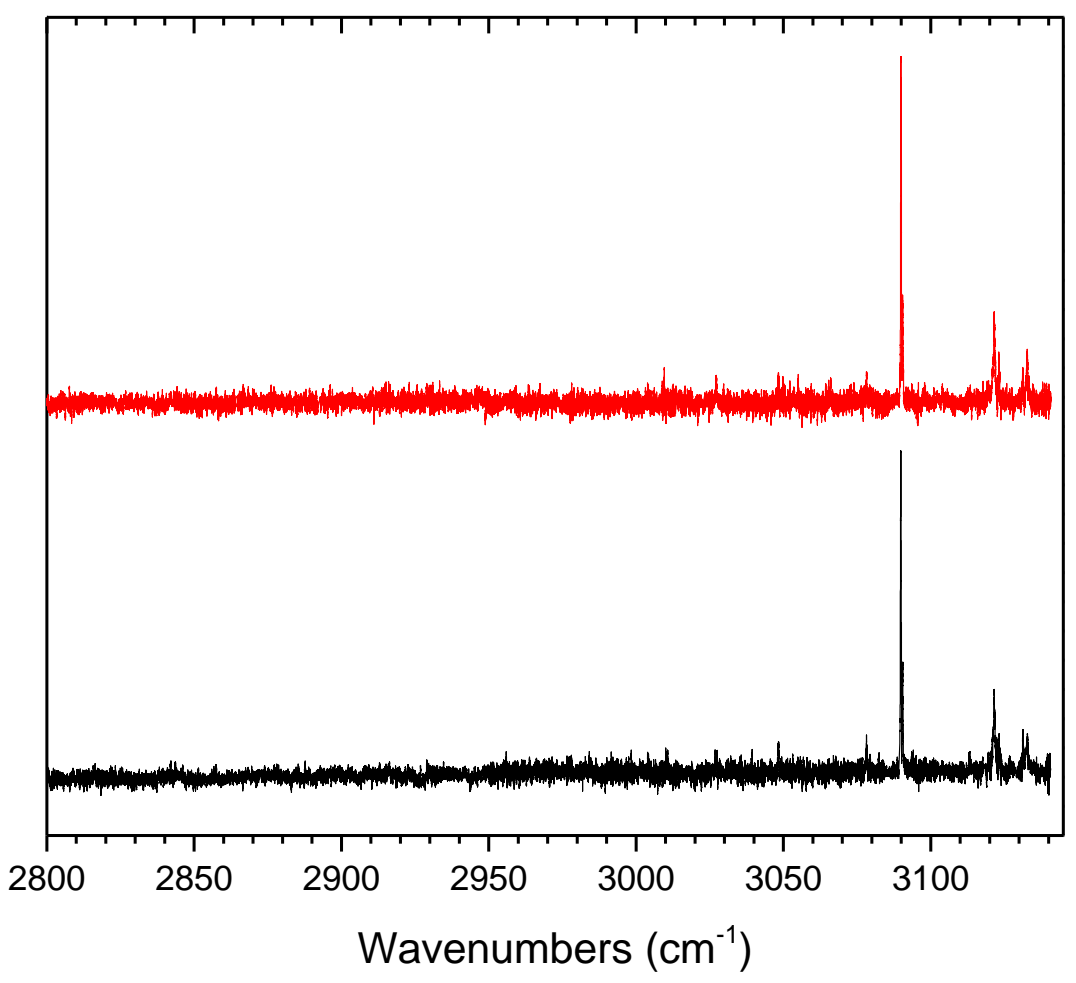

Figure S3. Mass channel 63 u comparison for cold pyro (black) and hot pyro (red). No obvious difference is observed between the two spectra aside from very weak features near the signal to noise limit. 
Table S2. Symmetries, identities, and VPT2+K frequencies and intensities of vibrational transitions of 5-ethynylcyclopentadiene between 2800 and $3400 \mathrm{~cm}^{-1}$.

\begin{tabular}{|c|c|c|c|}
\hline$\Gamma$ & $v\left(\mathrm{~cm}^{-1}\right)$ & $\mathrm{I}(\mathrm{km} / \mathrm{mol})$ & Character \\
\hline$a^{\prime}$ & 3369.54 & 1.17 & $v_{5}+v_{8}$ \\
\hline$a^{\prime}$ & 3357.40 & 0.87 & $v_{8}+v_{9}+v_{10}$ \\
\hline$a^{\prime}$ & 3339.60 & 60.49 & $v_{1}$ \\
\hline$a^{\prime}$ & 3175.97 & 0.34 & $2 v_{22}$ \\
\hline$a^{\prime}$ & 3111.09 & 3.10 & $v_{2}$ \\
\hline$a^{\prime}$ & 3091.89 & 4.70 & $v_{3}$ \\
\hline$a^{\prime}$ & 3015.20 & 0.20 & $2 v_{6}$ \\
\hline$a^{\prime}$ & 2872.41 & 0.14 & $v_{6}+v_{7}$ \\
\hline$a^{\prime}$ & 2871.00 & 2.22 & $v_{4}$ \\
\hline$a^{\prime}$ & 2854.30 & 0.26 & $v_{22}+v_{23}$ \\
\hline$a^{\prime \prime}$ & 3130.96 & 4.00 & $v_{20}$ \\
\hline$a^{\prime \prime}$ & 3122.36 & 0.18 & $v_{22}+v_{10}+v_{17}$ \\
\hline$a^{\prime \prime}$ & 3085.34 & 3.53 & $v_{21}$ \\
\hline$a^{\prime \prime}$ & 3064.32 & 2.33 & $v_{6}+v_{22}$ \\
\hline$a^{\prime \prime}$ & 2937.71 & 0.26 & $v_{7}+v_{22}$ \\
\hline
\end{tabular}

Table S3. Symmetries, identities, and VPT2+K frequencies and intensities of vibrational transitions of 1-ethynylcyclopentadiene between 2800 and $3400 \mathrm{~cm}^{-1}$.

\begin{tabular}{|c|c|c|c|}
\hline$\Gamma$ & $v\left(\mathrm{~cm}^{-1}\right)$ & $\mathrm{I}(\mathrm{km} / \mathrm{mol})$ & Character \\
\hline$a^{\prime}$ & 3379.96 & 0.42 & $v_{6}+v_{11}$ \\
\hline$a^{\prime}$ & 3340.42 & 27.29 & $v_{6}+v_{12}$ \\
\hline$a^{\prime}$ & 3335.26 & 48.41 & $v_{1}$ \\
\hline$a^{\prime}$ & 3164.56 & 0.62 & $2 v_{7}$ \\
\hline$a^{\prime}$ & 3119.96 & 5.09 & $v_{2}$ \\
\hline$a^{\prime}$ & 3092.28 & 5.53 & $v_{3}$ \\
\hline$a^{\prime}$ & 3085.64 & 4.27 & $v_{4}$ \\
\hline$a^{\prime}$ & 3056.27 & 1.77 & $v_{7}+v_{8}$ \\
\hline$a^{\prime}$ & 2996.38 & 0.23 & $2 v_{8}$ \\
\hline$a^{\prime}$ & 2922.38 & 0.19 & $v_{7}+v_{10}$ \\
\hline$a^{\prime}$ & 2911.07 & 3.10 & $v_{5}$ \\
\hline$a^{\prime}$ & 2844.86 & 0.15 & $v_{8}+v_{10}$ \\
\hline$a^{\prime}$ & 2805.10 & 0.12 & $v_{7}+v_{12}$ \\
\hline$a^{\prime \prime}$ & 2926.60 & 2.85 & $v_{23}$ \\
\hline
\end{tabular}


Table S4. Symmetries, identities, and VPT2+K frequencies and intensities of vibrational transitions of 2-ethynylcyclopentadiene between 2800 and $3400 \mathrm{~cm}^{-1}$.

\begin{tabular}{|c|c|c|c|}
\hline$\Gamma$ & $v\left(\mathrm{~cm}^{-1}\right)$ & $\mathrm{I}(\mathrm{km} / \mathrm{mol})$ & Character \\
\hline$a^{\prime}$ & 3377.49 & 0.32 & $v_{6}+v_{11}$ \\
\hline$a^{\prime}$ & 3337.28 & 68.60 & $v_{1}$ \\
\hline$a^{\prime}$ & 3172.07 & 0.21 & $2 v_{7}$ \\
\hline$a^{\prime}$ & 3130.37 & 2.53 & $v_{2}$ \\
\hline$a^{\prime}$ & 3114.84 & 0.27 & $v_{8}+v_{26}+v_{28}$ \\
\hline$a^{\prime}$ & 3100.55 & 3.16 & $v_{3}$ \\
\hline$a^{\prime}$ & 3089.59 & 1.25 & $v_{4}$ \\
\hline$a^{\prime}$ & 3073.97 & 1.28 & $v_{7}+v_{8}$ \\
\hline$a^{\prime}$ & 3028.89 & 0.47 & $2 v_{8}$ \\
\hline$a^{\prime}$ & 2904.84 & 0.65 & $v_{8}+v_{9}$ \\
\hline$a^{\prime}$ & 2901.10 & 6.48 & $v_{5}$ \\
\hline$a^{\prime \prime}$ & 2912.30 & 4.67 & $v_{23}$ \\
\hline
\end{tabular}

